\title{
Oral bisphosphonate use reduces cardiovascular events in a cohort of Danish patients referred for bone mineral density
}

Alexander J. Rodriguez, ${ }^{1,2}$, Martin T. Ernst', Mads $\mathrm{Nybo}^{3}$, Daniel Prieto-Alhambra ${ }^{4,5}$, Peter R. Ebeling ${ }^{1,6}$, Anne Pernille Hermann7, Bo Abrahamsen2,4,8

1. Bone and Muscle Health Research Group, Department of Medicine, School of Clinical Sciences, Monash University, Clayton, Victoria 3168, Australia

2. OPEN-Open Patient Data Explorative Network, Institute of Clinical Research, University of Southern Denmark, 5000, Odense, Denmark

3. Department of Clinical Biochemistry and Pharmacology, Odense University Hospital, 5000 Odense, Denmark

4. Pharmaco- and Device Epidemiology, Centre for Statistics in Medicine, Nuffield Department of Orthopaedics, Rheumatology and Musculoskeletal Sciences, University of Oxford, Oxford, UK.

5. Grup de Recerca en Malalties Prevalents de l'Aparell Locomotor (GREMPAL) Research Group and CIBERFes, University Institute for Primary Care Research (IDIAP) Jordi Gol, Universitat Autonoma de Barcelona and Instituto de Salud Carlos III, Barcelona, Spain

6. Australian Institute for Musculoskeletal Science, Melbourne Medical School-Western Campus, Faculty of Medicine, Dentistry and Health Sciences, The University of Melbourne, Melbourne, Australia

7. Department of Endocrinology and Metabolism, Odense University Hospital, 5000 Odense, Denmark.

8. Department of Medicine, Holbæk Hospital, Holbæk, Denmark

Running title: Cardiovascular safety of oral bisphosphonates

Corresponding author:

Dr. Alexander Rodríguez

Bone and Muscle Health Research Group, Department of Medicine, School of Clinical Sciences at Monash Health, Faculty of Medicine, Nursing and Health Sciences, Monash University

Monash Medical Centre, 246 Clayton Road,

Clayton, Victoria,

Australia 3168.

Ph: + 61385722919 Fax: + 61395946495 Email: alexander.rodriguez@monash.edu

Word count: 3507

Abstract word count: 233

\section{Conflict of interest}

All authors have completed the ICJME uniform disclosure form at www.icmje.org/coi disclosure.pdf. AJR was supported by the Christine \& TJ Martin Research Travel Grant from the Australia New Zealand Bone and Mineral Society, the Barbara Mawer Travelling Fellowship from the Bone Research Society, an Australian Government Research Training Stipend and Monash University. AJR declares no financial relationships with any organisations that might have an interest in the submitted work in the previous three years; no other relationships or activities that could appear to have influenced the submitted work. PRE declares research funding and honoraria from Amgen. All other authors declare: no support from any organisation for the submitted work; no financial relationships with any organisations that might have an interest in the submitted work in the previous three years; no other relationships or activities that could appear to have influenced the submitted work. 


\section{What is already known on this topic?}

- The effects of oral bisphosphonate treatment on cardiovascular outcomes are unknown, with analyses of secondary outcomes of previous randomized controlled trials (RCTs) reporting conflicting results

- However, RCT participants are rarely representative of real world patient populations. Observational data may therefore also guide clinical decision making, but previous observational reports have not matched individuals a priori or investigated prognostic factors for cardiovascular events.

\section{What this study adds}

- In this cohort of over 2000 Danish oral bisphosphonate users matched to highly-selected controls, all sharing a clinical indication for bone mineral density (BMD) assessment; oral bisphosphonate use was associated with an approximate $33 \%$ reduced risk of cardiovascular events.

- Adjustment for BMD did not alter outcomes. Pre-defined sensitivity analyses and positive and negative control outcomes added robustness.

- Our data support reports of a reduction of cardiovascular events seen in some RCTs of bisphosphonates; however, our study design makes these data transferable to real-world clinical practice 


\section{Abstract}

Context: The cardiovascular (CV) safety of oral bisphosphonates (oBP) is uncertain.

Objective: Determine the risk of CV events in oBP users referred for bone mineral density (BMD) testing compared with matched controls.

Design: Cohort study.

Setting: Danish national prescription registry enriched with local hospital data from Odense.

Participants: Individuals aged $\geq 45$ years referred for BMD testing.

Exposure: Oral BP.

Outcomes: Hospitalization for any CV event. Secondary study outcomes were specific CV events. Negative (inguinal hernia surgery and ingrown toenail) and positive (fragility fracture) control outcomes assessed systemic bias. Cox proportional hazards models were fitted to estimate hazard ratios (HR) [95\% confidence intervals].

Results: There were 2,565 oBP users ( $82.6 \%$ women) and 4,568 (82.3\% women) propensity scorematched controls. Alendronate accounted for $96 \%$ of oBP prescription. A total of $406(15.8 \%)$ $\mathrm{CV}$ events occurred in oBP users (rate $=73.48$ [66.67 to 80.98]); and $837(18.3 \%$ ) events in controls (rate $=104.73$ [97.87 to 112.07]) with an adjusted HR of 0.68 [0.60 to 0.77]. Additional adjustment for BMD did not attenuate estimates [HR of 0.67 (0.58 to 0.78)]. Similar results were seen for secondary outcomes where risk reductions were seen regarding atrial fibrillation, stroke, heart failure and aneurysms. Positive and negative control outcome analyses identified minimal residual confounding.

Conclusion: Oral BP users experienced a 33\% reduced risk of CV events. This observational realworld study adds to a growing body of evidence for cardio-protection by oBP that warrants testing in a randomized setting. 


\section{Introduction}

2 Osteoporosis describes the progressive loss of bone mass and deterioration in bone 3 microarchitecture leading to skeletal fragility and an increased susceptibility to fracture. 4 Osteoporosis or low bone mass does not exist in isolation, but often co-exists with other chronic 5 diseases, particularly cardiovascular [CV] diseases $(1,2)$. Awareness of the need for management of 6 CV risk factors alongside fracture risk is growing, given multiple reports of increased CV mortality 7 following osteoporotic fragility fracture (3-5).

8 Critically, low bone mineral density [BMD], which predisposes to fracture, has been positively 9 associated with an increased risk of CV diseases including high blood pressure, vascular calcification, stroke and myocardial infarction [MI (6-8)]. Previous randomized controlled trials (RCTs) of zoledronic acid demonstrated either trends towards reduced CV mortality (9) ${ }^{9}$ or a reduced risk of MI, suggesting a biological link between the skeleton and the CV system, as well as a protective role for the bisphosphonates. Two recent meta-analyses have examined mortality and CV effects of BPs with uncertain findings $(10,11)$. Other anti-osteoporosis drug RCTs have demonstrated trends towards an increase in adjudicated CV events, as seen with romosozumab, strontium ranelate and odanacatib; for which use of the latter two drugs were ceased owing to CV safety concerns (12-15). Interestingly, the romosozumab RCT included alendronate as the active comparator, which some have suggested reduces CV events, leading to speculation that the elevation in $\mathrm{CV}$ events in the romososzumab arm in this trial may have been artificial, as it was not seen in the placebo-controlled RCT $[(14,16)$.

Participants in RCTs are rarely representative of the real-world patient populations typical of clinical practice. As such, large observational studies comparing treated patients directly with realworld controls are an important investigative tool to understand CV risks. Such observational data may complement data from RCTs $(17,18)$. Given the hypothesized link between the skeleton and the $\mathrm{CV}$ system; consideration of both $\mathrm{CV}$ risk factors and $\mathrm{BMD}$ are critical when interpreting data from cohorts of patients with osteoporosis.

In this regard, a Danish study including typical bisphosphonate (BP) users, the use of any BP was associated with an approximate $40 \%$ increased risk of incident heart failure relative to controls (19). Another Danish registry study showed an approximate $20 \%$ increased risk of atrial fibrillation in BP-treated fracture patients after multiple adjustments (20). Combining Danish and Swedish health registries, compared with oral BP, intravenous zoledronic acid was associated with an increased risk of heart failure(21). By contrast, recent data from Hong Kong examining hip fracture patients showed alendronate use was associated with both reduced one-year CV mortality and incident MI (22). Following a 10-year follow-up, these risk reductions remained significant, but were attenuated. Incident stroke risk was reduced after one year when considering all nitrogen containing BPs and this was also maintained out to 10 years; but alendronate was only associated with reduced stroke risk at five years and 10 years.

Observational studies on this topic have been limited in their investigation of prognostic factors for CV events. Further, they did not include individuals matched on indication meaning they may have had an inherent design bias. We therefore aimed to determine, in a nationally representative sample from Denmark, including oral BP (oBP) users and non-users who also had BMD assessments, the risk of incident $\mathrm{CV}$ disease associated with oBP use. 


\section{Methods}

\section{$46 \quad$ Data sources}

47 This study is an analysis of the Odense Bisphosphonate Safety Study (OBSS) and conducted in 48 accordance with STROBE guidelines (23). The OBSS is a prospective cohort of patients examined 49 for osteoporosis undergoing BMD assessment using dual-energy x-ray absorptiometry [DXA] at 50 Odense University Hospital (OUH, Odense, Denmark) (24). The study was conducted under the 51 auspices of the OUH Open Patient Data Explorative Network (OPEN) framework (Statistics 52 Denmark project approval \#5079). Unlike randomly selected population controls, all individuals in the cohort shared a clinical indication for DXA. The cohort is enriched with additional clinical and biochemical information from local and national health registries. Specifically, clinical information on demographics, anthropometry, renal function (within one year of the index date) estimated by glomerular filtration rate (eGFR) (25) were obtained from local hospital records. Prior fractures, Charlson co-morbidity index (for co-morbidities in the previous year), prescription history and hospitalizations were obtained from national health registries.

$\underline{\text { Study participants }}$

60 Individuals were considered exposed (oBP user) if they filled at least one prescription for an oBP 61 (representing new users) from 1 January 2003 to 31 December 2014 had areal BMD (hip and spine) 62 determined by DXA within two years of the prescription. Anatomical Therapeutic Classification 63 codes used to identify oBP users were: M05BA01-08 and M05BB01-09. Exclusion criteria were individuals aged $<45$ years at index date; first oBP prescription was intravenous BP; use of other bone active medications (except menopausal hormone replacement therapy) that are not oBPs;

66 Paget's disease of bone; malignancies; migration out of Denmark before the index date; death at index date itself. All other persons meeting the study criteria were evaluated as potential nonexposed controls (non-users) until the time of censoring or beginning an oBP treatment. This population forms the pool of potential control persons who were tabulated for the before matching' scenario and used in the evaluation of the subsequent matching.

Matching

To account for non-random assignment of oBP treatment, we used a propensity score matching procedure in a ratio of 1:2 (oBP:controls). The propensity score represents the likelihood of being assigned to treatment given the set of measured confounders present.

$\underline{\text { Sample size calculation }}$

Based off expected CV events from Danish and Swedish national health registries data (assuming $\alpha$ of $5 \%$ and $\beta$ of $20 \%$ ), the number of exposed and non-exposed needed to determine clinically significant differences in outcomes are 2,264 and 4,528 respectively $(21,26)$.

\section{Follow-up and outcome analysis}

Analyses were performed in an intention-to-treat manner considering an oBP user as always being exposed upon first prescription. We compared oBP users with propensity score matched nonusers (propensity score matched sub-cohort). The primary study outcome of this study was hospitalization for any CV disease. Secondary study outcomes included cause-specific incident hospitalization for CV disease, specifically: atrial fibrillation or flutter, MI, cardiac arrhythmia, heart 
failure, all stroke, ischaemic stroke, atherosclerotic vascular disease, aneurysms and peripheral artery disease. Exploratory outcomes included mortality due to any CV disease, mortality due to MI, stroke and all causes. We aimed to identify any systematic bias by including both positive control outcomes (osteoporotic fractures at any site, anticipated to be reduced by oBP treatment) and negative control outcomes (ingrown toenails and inguinal hernia surgery, not expected to be influenced by oBP treatment). All outcomes were defined by ICD-10 codes and are shown in the supplementary documents (27). Follow-up began on the date of first oBP prescription.

To control for immortal time bias, matches were made in a time-dependant manner. In this scenario, the index date of the oBP user is inherited by their matched non-user. Individuals were censored on the date of study outcome, uptake of non-oral BP anti-osteoporosis medications, death, migration out of Denmark or 31 December 2014, whichever came first.

For secondary study outcomes, the time to event was specific to that specific CV hospitalization. For example, an individual first records atrial fibrillation and then suffers a subsequent MI. For the primary outcome, the time to atrial fibrillation is used as it occurred first. For the MI-specific analysis, the time to MI is used ignoring that an atrial fibrillation event occurred prior to the MI. Thus, survival time was calculated for each secondary event independently. A Cox proportional hazards model was then fitted to estimate survival time between groups. Violation of the proportional hazard assumption was checked by visual inspection of c-log-log plots. Violation was evident and thus, oBP use was treated as a time varying co-variate. Survival time for oBP users was split into two separate records to account for time initially non-exposed and then subsequently exposed. Therefore, for individuals who began as non-exposed in the analysis window, they were censored at the date of oBP prescription and could then serve as an exposed individual (i.e. they contribute risk time as both a control and an oBP user).

In the propensity score-matched analysis, models were fitted with oBP use; propensity score and finally, propensity score and all unbalanced covariates based on propensity score. All analyses were subsequently performed again on a per-protocol basis where oBP users were then additionally censored if there was period greater than 60 days of not filling a prescription during follow-up. To investigate the validty of our findings, we performed all analysis in a simple age- and sex-matched sub-cohort (27). All analyses were performed using Stata v15.1 (StataCorp, College Station, Texas, USA). Data were computed by AJR with checks performed by MTE, DPA and BA.

\section{Propensity score}

The propensity score was estimated using a multivariable logistic regression model including information on age, sex, BMI, Charlson co-morbidity index, prior fractures, number of medications, eGFR and previous hospitalization for a cardiovascular disease. Matches were made using a caliper width of 0.2 standard deviations. Those who failed to be matched were excluded from analysis (Figure 1). Because BMD is the indication for oBP initiation, it was not part of the matching algorithm and the results are presented with and without adjustment for BMD. To assess the quality of matching, the absolute standardized difference (St. Diff) in variables between oBP users and matched non-users were calculated. Any variable with an absolute standardized difference greater than 0.1 was considered unbalanced and adjustment was made for this variable in outcome analyses. Matching was achieved using the gmatch command (Mayo Clinic, 2003).

\section{Funding}


No external funding.

\section{Results}

131 Baseline characteristics

132 We identified 5,643 oBP users (80.9\% women) and 21,879 non-users ( $76.5 \%$ women). Alendronate

133 accounted for $96 \%$ of oBP prescriptions. Intravenous BP users were excluded. Oral BP users were

134 older, had greater BMI, a greater number of co-morbidities and medications, greater prevalence of

135 mild to severe renal impairment, poorer bone health and a higher prevalence of previous fractures

136 [Table 1]. The propensity score matched sub-cohort comprised 2,565 oBP users (82.6\% women)

137 and 4,568 non-users (82.3\% women). Balance was achieved on all matching variables apart from

138 fracture history (oBP users: $29.4 \%$ vs. non-users: $24.8 \%$; $\mathrm{SD}=0.110)$, lumbar spine aBMD (0.796

$139 \mathrm{~g} / \mathrm{cm}^{2}[0.732,0.881]$ vs. $0.852 \mathrm{~g} / \mathrm{cm}^{2}[0.752,0.964]$; $\left.\mathrm{SD}=0.355\right)$, lumbar spine T-score $(-2.4$ [-2.9,

$140-1.7]$ vs. $-1.7[-2.7,-0.7] ; \mathrm{SD}=0.437)$ and prevalence of osteoporosis based on lumbar spine $\mathrm{T}$-score

$141 \quad(45.7 \%$ vs. $28.9 \%$; $S D=0.406)$ [Table 1$]$.

142

143 Event analysis

$144 \quad$ Propensity score-matched sub-cohort

145 For the primary outcome, there were $406(15.8 \%)$ events in oBP users [rate $=73.48$ (66.67 to 146 80.98)] and $837(18.3 \%)$ in non-users [rate=104.73 (97.87 to 112.07)] [Table 2]. On Cox regression, 147 oBP use was associated with a $26 \%$ reduced risk of $\mathrm{CV}$ events [0.74; 0.65 to 0.83]. Further 148 adjustment for the remaining unbalanced variables (lumbar spine BMD, osteoporosis in the spine 149 and prior fracture at any site) did not materially alter this effect estimate [0.67; 0.58 to 0.78 ] [Figure 150 2].This situation was replicated regarding the outcomes of atrial fibrillation, heart failure, stroke, ischaemic stroke, atherosclerotic vascular disease and aneurysm [Table 3].

As a positive control outcome, we analysed the effect of oBP use on osteoporotic fractures. Similarly, there were $247(9.6 \%)$ fractures in oBP users and $630(13.8 \%)$ fractures in propensity score-matched non-users with an adjusted HR of 0.26 [0.22 to 0.33$]$.

With respect to negative control outcomes, compared to propensity-score matched non-users, no association was evident regarding inguinal hernia surgery [0.94 (0.67 to 1.32)] or ingrown toenails [8,73 (0.77 to 98.11)], suggesting minimal residual confounding in the propensity score analyses. Similar results were seen when analyses were repeated on a per-protocol basis [Table 4].

163 In the propensity score-matched sub-cohort, oBP use was only associated reduced risk of CV or all-cause mortality [Table 3]. All the above analyses were repeated on a per-protocol basis, where oBP use was additionally censored when a prescription was not filled after 60 days [Table 3 \&

166 Supplementary Table 2]. An age- and sex-matched sub-cohort comprised 3,955 oBP users (80.3\% women) and 6,867 non-users (79.3\% women). For the primary outcome, there were $737(18.6 \%)$ events in oBP users [rate per 1000 person-years: 86.44 (80.41 to 92.91)] and 1,706 (24.8\%) in nonusers [rate $=121.92$ (116.27 to 127.84)]. In a Cox regression, oBP use was associated with a reduced risk of $\mathrm{CV}$ events [0.74; 0.67 to 0.80$]$. In an adjusted model controlling for the variables included in the propensity score model), the magnitude of risk reduction increased [0.56; 0.50 to 0.64$]$ and 
additional adjustment for femoral neck BMD [0.56; 0.49 to 0.63$]$ did not alter the risk estimate.

173 This was similarly the case for other outcomes, however, oBP use was associated with a reduction

174 risk inguinal hernia surgery [HR: 0.63 (0.46 to 0.85)] suggesting some systemic bias in the age- and 175 sex-matched sub-cohort analysis. All exploratory data is found in a data repository(27).

\section{Discussion}

\section{$\underline{\text { Highlight }}$}

180 In this nationally representative cohort of Danish individuals assessed for BMD, oBP use was associated with a $33 \%$ reduction in risk of $\mathrm{CV}$ events. We further explored the validity of this association through multiple approaches, including by additional adjustment for BMD, which did not alter this outcome. In other words, ensuring both users and non-users came from a population with a clinical indication for BMD measurement helped to define a control population that was more appropriate to an osteoporosis treatment scenario than relying on randomly selected population controls, which would tend to have lower overall comorbidity. Positive and negative control outcomes excluded residual confounding from our analyses. Oral BP use was associated with an anticipated reduction in fracture rate, but was not associated with inguinal hernia repair or ingrown toenails, both of which would be unanticipated benefits of oBP use. Overall, our findings indicate that real-world oBP users may gain some cardio-protective benefit from oBP, particularly alendronate which accounted for $96 \%$ of prescribed oBPs in this cohort.

\section{What this study adds}

194 Our study represents a significant contribution to the literature on oBP use and CV risk. First, as participants in RCTs are rarely representative of typical oBP users in clinical practice, this cohort of individuals represents a unique real-world investigation of oBP effects on CV events. Second, as no BP RCT has examined CV events or mortality as a primary study outcome, practice guidelines need to also rely on findings from observational studies. In this regard, the OBSS was specifically designed to examine such outcomes. Third, unlike usual population controls from other cohorts, the non-users in the OBSS are all matched on a clinical indication for bone densitometry by DXA. However, across all analyses, adjustment for baseline BMD did not materially alter any outcome. This is potentially significant as the protective benefit of BP may act through mechanisms independent of bone mass. Previous cohort data have suggested that nitrogen containing BPs (such as alendronate) may have a role in the mevalonate pathway, for which other medications influencing this pathway, such as statins, have proven cardio-protective benefits(28). Additionally, BPs are taken up by macrophages and have effects on cytokine release by T cells, which may modulate inflammation, an important precedent to CV disease (29).

210 In ageing populations, individuals present with multiple co-morbidities. Thus, from a clinical 211 practice perspective, increasing attention has been given to the safety of medications in the setting 212 of co-existing chronic diseases. This is particularly relevant to medications used in the long-term 213 (such as oBPs) as such individuals are likely to accumulate poorer outcomes over time. A recent 214 Italian working group proposed that BP use in patients with $\mathrm{CV}$ conditions was potentially 215 inappropriate representing an area for future research (30). This recommendation was borne out 
216 of reports from RCTs, as well as from observational cohorts, suggesting BP use increased atrial 217 fibrillation and cerebrovascular events $(31,32)$. These risks predominantly concern zoledronic acid 218 use and an increase in atrial fibrillation and flutter in the HORIZON Pivotal Fracture Trial, in 219 which there was a greater incidence of serious atrial fibrillation in the treatment arm (50 (1.3\%) vs. $22020(0.5 \%)$ patients; $\mathrm{p}<0.001)$. However, there was no such increase in an older and more fragile 221 hip fracture population in the HORIZON Recurrent Fracture Trial (33). Zoledronic acid also lead 222 to a reduction in stroke death in osteopenic women compared with placebo [Odds ratio: 0.14; $95 \%$

223 CI: 0.01 to 0.92$](34)$. Interestingly, investigators from the alendronate-controlled trial of 224 romosozumab explained that their findings of decreased events in the alendronate arm was most 225 likely due to chance (35) This important controversy, which has large clinical implications for 226 management osteoporosis in patients with comorbid conditions highlights the need for supporting 227 data from observational cohort studies that can evaluate further the hypothesis that BP reduce CV 228 events in a real-world population with a broader range of comorbid conditions than in clinical 229 trials.

231 Our findings corroborate observational reports of cardio-protective effects of BP use (21). In 232 patients attending a Scottish fracture liaison service, BP use was associated with a $21 \%$ reduced 233 mortality risk [0.79; 0.64 to 0.97], even after adjustment for subsequent fracture compared with 234 calcium and vitamin D treatment (36). In the present study, we showed a similar 50-62\% reduced 235 risk of CV mortality(22). However, in a bi-national study of oral BP users in patients with moderate 236 to severely impaired renal function, there was no CV mortality benefit (37). It is important to note 237 that our study was only powered to detect clinically important differences in CV events. Our 238 findings also support claims of a suppression of CV events recently seen in RCTs involving 239 romosozumab, which included alendronate as an active comparator(14).

\section{Strengths}

242 This study had several strengths. First, all patients and controls had BMD measurements meaning all participants in the study had a clinical indication for bone densitometry which is unlike any study to date. Our strategy differs from previous observational studies investigating the CV risks of oBP therapy, whereby the oBP user groups were drawn from patients who had fractured or had underlying co-morbidities $(20,22,37)$. Therefore, this meant that by design, users and non-users in the present study were better matched a priori than in previous observational studies on a similar topic. Second, our study had several measures of internal validity. We employed two sets of analyses: users versus (i) propensity score-matched controls and; (ii) age- and sex-matched controls. We assessed positive and negative control outcomes to investigate systemic bias, for which these analyses suggested there was minimal bias. We acknowledge however, that the study was not powered to detect differences in control outcomes, but they serve as an important examination of bias. Overall, our analysis represents comparisons among both real-world patients on oBPs and highly matched control patients which adds robustness to conclusions.

This study was limited by its observational design. Although we employed several techniques to minimise confounding and bias, we cannot rule out the potential for unmeasured confounding and residual bias to influence outcomes. We observed large reductions in osteoporotic fractures (positive control outcome). It is important to note that this study was not a trial emulation study 
and HR are not expected to mirror those seen in RCTs. Indeed, large effect sizes are sometimes seen in exploratory secondary outcomes in RCTs. Although we employed propensity score matching to control for baseline confounding (like randomization in an RCT) residual confounding does remain. Further, there could be some healthy adherer effect which is not unique to observational studies and is also seen in RCTs. Unlike previous studies in this area, we had access to clinical information such as renal function and BMD, which strengthened our investigation of prognostic factors. However, we did not have access to information on lifestyle factors such as smoking and alcohol use which are known to influence CV events. The study, whilst being nationally representative of Denmark, may not be generalizable to other populations at risk of osteoporosis and CV disease. Most oBP users (96\%) were prescribed alendronate in this study, thus we are unable to fully attribute the findings solely to alendronate. Replication of this study in settings with a greater uptake of other oBP such as risedronate and oral ibandronate are warranted.

\section{Lessons for future research}

276 Adjustment in multivariable models for baseline BMD did not alter effect estimates. This finding 277 is in contrast to reports that BMD is a predictor of CV disease and mortality (5,38-40). Our finding 278 suggests that, although lumbar spine BMD remained unbalanced after matching which may result 279 in residual confounding; claims/electronic medical records databases may still provide a robust 280 platform to analyse CV risks as BMD did not influence model significance. Further understanding 281 of prognostic factors for $\mathrm{CV}$ events in the setting of osteoporosis is needed to identify specific 282 patient sub-groups most at risk and most likely to benefit from treatment (for example differing 283 associations in hip fracture patients and patients with renal impairment). Greater collaboration 284 amongst clinical specialities is warranted to manage apparent co-existing disease risks which may act synergistically to incur poorer outcomes(41).

\section{Conclusion}

In this nationally representative cohort of Danish oral BP users matched on a clinical indication for DXA assessment to non-users, there was a reduced risk of CV events. These data complement findings from BP RCTs showing a reduction in CV events. Additional adjustment for BMD did not alter outcomes, which questions the prognostic value of BMD on CV risk in the setting of 
296 AJR was involved in study design, conducted all analyses, tabulated data, ensured data integrity 297 and wrote the first draft. MTE provided statistical support and ensured data integrity. MD oversaw 298 ascertainment of biochemical data. DP-A provided advice on study design, provided statistical 299 support, provided clinical advice on data interpretation and advised on manuscript drafting. PRE 300 provided clinical advice on data interpretation and advised on manuscript drafting. PH oversaw 301 ascertainment of DXA information. BA is the guarantor of the project, provided the initial concept 302 for the project, provided advice on study design, provided statistical support, provided clinical 303 advice on data interpretation and advised on manuscript drafting. All authors reviewed the final 304 draft.

306 Institutional review board approval: This study was approved by the Odense University Hospital 307 (OUH) Open Patient Data Explorative Network (OPEN) review board (Statistics Denmark 308 project approval \#5079).

\section{Acknowledgements:}

311 The authors wish to acknowledge Dr. Nazmul Karim and Dr. Lucy Busija both of Monash 312 University for their assistance in checking and advising on statistical coding. 


\section{References:}

1. Sennerby U, Farahmand B, Ahlbom A, Ljunghall S, Michaelsson K. Cardiovascular diseases and future risk of hip fracture in women. Osteoporosis International 2007;18(10):1355-1362.

2. Sennerby U, Melhus H, Gedeborg R, Byberg L, Garmo H, Ahlbom A, Pedersen NL, Michaëlsson K. Cardiovascular Diseases and Risk of Hip Fracture. JAMA 2009;302(15):1666.

3. Bliuc D, Nguyen ND, Milch VE, Nguyen T v, Eisman JA, Center JR. Mortality Risk Associated With Low-Trauma Osteoporotic Fracture and Subsequent Fracture in Men and Women. JAMA 2009;301(5):513.

4. Bliuc D, Alarkawi D, Nguyen T v, Eisman JA, Center JR. Risk of Subsequent Fractures and Mortality in Elderly Women and Men with Fragility Fractures with and without Osteoporotic Bone Density: The Dubbo Osteoporosis Epidemiology Study. Journal of Bone and Mineral Research 2015;30(4):637-646.

5. Browner WS, Seeley DG, Vogt TM, Cummings SR. Non-trauma mortality in elderly women with low bone mineral density. Study of Osteoporotic Fractures Research Group. Lancet (London, England) 1991;338(8763):355-8.

6. Yang S, Nguyen ND, Center JR, Eisman JA, Nguyen T v. Association between hypertension and fragility fracture: a longitudinal study. Osteoporosis International 2014;25(1):97-103.

7. Mussolino ME, Madans JH, Gillum RF. Bone mineral density and mortality in women and men: the NHANES I epidemiologic follow-up study. Annals of epidemiology 2003;13(10):692-7.

8. Samelson EJ, Kiel DP, Broe KE, Zhang Y, Cupples LA, Hannan MT, Wilson PWF, Levy D, Williams SA, Vaccarino V. Metacarpal Cortical Area and Risk of Coronary Heart Disease: The Framingham Study. American Journal of Epidemiology 2004;159(6):589-595.

9. Colón-Emeric CS, Mesenbrink P, Lyles KW, Pieper CF, Boonen S, Delmas P, Eriksen EF, Magaziner J. Potential mediators of the mortality reduction with zoledronic acid after hip fracture. Journal of Bone and Mineral Research 2010;25(1):91-97.

10. Cummings SR, Lui LY, Eastell R, Allen IE. Association between Drug Treatments for Patients with Osteoporosis and Overall Mortality Rates: A Meta-analysis. JAMA Internal Medicine 2019;179(11):1491-1500.

11. Kim DH, Rogers JR, Fulchino LA, Kim CA, Solomon DH, Kim SC. Bisphosphonates and Risk of Cardiovascular Events: A Meta-Analysis. Pizzi C, ed. PLOS ONE 2015;10(4):e0122646.

12. Reginster JY. Cardiac concerns associated with strontium ranelate. Expert Opinion on Drug Safety 2014;13(9):1209-1213.

13. McClung MR, O'Donoghue ML, Papapoulos SE, Bone H, Langdahl B, Saag KG, Reid IR, Kiel DP, Cavallari I, Bonaca MP, Wiviott SD, de Villiers T, Ling X, Lippuner K, Nakamura T, Reginster JY, Rodriguez-Portales JA, Roux C, Zanchetta J, Zerbini CAF, Park JG, Im KA, Cange A, Grip LT, Heyden N, DaSilva C, Cohn D, Massaad R, Scott BB, Verbruggen N, Gurner D, Miller DL, Blair ML, Polis AB, Stoch SA, Santora A, Lombardi A, Leung AT, Kaufman KD, Sabatine MS, Mautalén CA, Man Z, Zanchetta JR, Magaril CH, Sambrook P, Geusens P, Goemaere S, Albergaria BH, Zerbini CA de F, Castro ML, Gregorio LH, Stoilov R, Borissova AMI, Hristozov KH, Temelkova NL, Daskalova IK, Kuzmanova SI, Yaneva-Bichovska D, Batalov AZ, Riedemann P, Rodriguez Portales JA, Tang H, Zhu hanmin, Zhang Z, Chao A, Hu Y, Liu Z, Lu J, Qiu M, 
Gao X, Zhang S, Xu L, Xia W, Liao E, Yang W, Wu W, Dai K, Hu R, Jaller JJ, Cabal F, Molina JF, Cure Cure CA, Yupanqui-Lozno H, Chalem P, Londono J, Abello M, Tobias ED, Otero W, Nikolic T, Miskic B, Stepan J, Vyskocil V, Novosad L, Slesinger J, Novosad P, Vlckova E, Bortlik L, Dokoupilova E, Hala T, Jensen JEB, Brixen KT, Langdahl BL, Schwarz P, Eskildsen PC, Eiken PA, Hermann AP, Gram J, Schou MB, Alexandersen P, Nedergaard B, Mejía DM, Estrella De Henriquez L, Páez N, Velazco C, Valter I, Vahula KL, Kull I, Maasalu K, Chapurlat R, Fardellone P, Benhamou CL, Weryha G, Herkt V, Martz R, Nischik R, Spieler W, Contzen C, Felsenberg D, Frieling I, Frahm E, Briones H, Sandoval B, Barrios P, García A, Avendaño C, González M, Guerra J, Tuna M, Díaz OM, Samayoa E, López E, Barrera JR, Palencia M, Cifuentes M, Alvarado G, López M, Chavez N, Haase F, Rivera R, González C, Tan K, Leung PC, Mandalam S, Pitale SU, Bantwal G, Ammini AC, Shaikh SSA, Kanakatte Mylariah PK, Dharmalingam M, Mukhopadhyay S, Jain A, Singh P, Shetty N, Sathyanarayana SS, Shah N, Chadha MD, Bhandankar R, Velayutham K, Marwah S, John M, Sahay RK, Adami S, Nuti R, Bianchi G, Brandi ML, Minisola S, Fiore CE, Rubinacci A, Miyajima H, Yamane H, Nakatani Y, Okamoto S, Kuroda K, Fujimori M, Itabashi A, Katayama K, Nakajo S, Somekawa Y, Ohsawa Y, Tajima W, Mizuno K, Mori S, Kanabuchi T, Hashizume H, Oka N, Hamada K, Yamaguchi M, Hirahara F, Atobe M, Ohtake Y, Ichikawa S, Onishi T, Matsumoto K, Nakamura T, Shirasawa E, Katayama K, Takahashi M, Oguma T, Matsui H, Katoh Y, Shigenobu K, Onishi T, Shibukawa M, Ikeda S, Osaka K, Kanda R, Inobe Y, Shigenobu M, Hasegawa M, Yamaji T, Miyazaki Y, Ito T, Nakamura E, Nagai S, Lim SK, Chung YS, Shin CS, Min YK, Kim GS, Yoon HK, Kang M il, Yang KH, Park HM, Kim IJ, Chung DJ, Chung HY, Jaundzeikare S, Andersone D, Medne A, Yaghi Y, Alekna V, Kasiulevicius V, Purtokaite - Labutiniene I, Krasauskiene A, Varanaviciene J, Basijokiene V, Abraitiene A, Radzeviciene L, Walliser J, García Hernández PA, Araujo MF, Avila Armengol HE, de la Peña P, Tamayo J, Zazueta B, Cons F, Gilchrist NL, Reid IR, Leikis R, Jones P, Singh JGP, Halse JI, Syversen U, Høivik HO, Øfjord ES, Gulseth HC, Elle S, Norheim PD, Calvo Quiroz AA, Cesar Augusto PA, León Portocarrero MG, Vidal Neira LF, Chavez J, Garro Barrera B, Kuroiwa Sampei R, Luis Fernando B v., Oquelis Cabredo R, Castillo S, Morales AMG, Tan PP, Leagogo LAC, Wang EH, Li-Yu JT, Sawicki AZ, Stasiuk B, Kania G, Lorenc R, Sidorowicz-Bialynicka A, Szczepanski L, Franek E, Filip R, Sekula J, Blicharski T, Leszczynski P, Sewerynek E, Miazgowski T, Milewicz A, Dabrowska M, Romaszko J, Pluskiewicz W, Wojnowski L, Codreanu C, Bolosiu H, Ionescu R, Zosin I, Macovei L, Bojinca M, Radulescu F, Pop S, Sarbu A, Benevolenskaya LI, Nasonov EL, Rozhinskaya LY, Oganov RG, Rodionova SS, Shlyakhto EV, Trofimov V, Zotkin EG, Zazerskaya IE, Grineva EN, Ershova O, Lesnyak O, Ostroumova OD, Malichenko SB, Pikhlak EG, Pilyaev VG, Raskina T, Zonova E v., Shirinsky VS, Dimic AN, Cobeljic G, Vujovic S, Ellis GC, Lipschitz S, de Villiers TJ, de Weerd AJ, Vally T, Trinder Y, Coetsee JL, Davis CP, Nayiager S, Hough FS, Oelofse LF, van der Walt E, Lombaard JJ, Blignaut S, Govind U, Fouche LF, Kruger DS, Dalmeyer JP, Ferreira MM, Escudero-Contreras A, Muñoz Torres M, Hawkins Carranza F, Perez Castrillon JL, García Meijide JA, Jodar Gimeno E, Palacios Gil-Antuñana S, de Teresa Parreno L, Martín Mola E, Alvarez Sanchez C, Tsai KS, Tu S te, Chen JF, Lee OKS, Hsu WW, Grygorieva NV, Povoroznyuk VV, Korzh MO, Loskutov OL, Chukov AB, Sarmiento R, Thomas H, Donnachie H, Pavel-Knox I, Shaw H, Hassanin H, Abdulhakim EEA, 
Savani N, Bachmann GA, Barrett-Connor E, Binkley NC, Bone HG, Brandon DM, Checketts DD, Fraser NJ, Watts NB, Geller SA, Gimbel JS, Greenwald MW, Holt PA, Johnston CC, Fang C, Klashman DJ, Lewiecki EM, Lowenstein MB, McClung MR, Nattrass SM, Odio A, Levengood J, Romaguera J, Sebai MB, Snyder B, Kutner ME, Streja D, Schwartz EP, Christiansen MG. Odanacatib for the treatment of postmenopausal osteoporosis: results of the LOFT multicentre, randomised, double-blind, placebo-controlled trial and LOFT Extension study. The Lancet Diabetes and Endocrinology 2019;7(12):899-911.

14. Saag KG, Petersen J, Brandi ML, Karaplis AC, Lorentzon M, Thomas T, Maddox J, Fan M, Meisner PD, Grauer A. Romosozumab or Alendronate for Fracture Prevention in Women with Osteoporosis. New England Journal of Medicine 2017;377(15):1417-1427.

15. Saag KG, Petersen J, Brandi ML, Karaplis AC, Lorentzon M, Thomas T, Maddox J, Fan M, Meisner PD, Grauer A. A Randomized Alendronate-Controlled Trial of Romosozumab: Results of the Phase 3 Active-Controlled Fracture Study in Postmenopausal Women with Osteoporosis at High Risk - ACR Meeting Abstracts. Available at: https://acrabstracts.org/abstract/a-randomized-alendronate-controlledtrial-of-romosozumab-results-of-the-phase-3-active-controlled-fracture-study-inpostmenopausal-women-with-osteoporosis-at-high-risk/. Accessed February 20, 2020.

16. Cosman F, Crittenden DB, Adachi JD, Binkley N, Czerwinski E, Ferrari S, Hofbauer LC, Lau E, Lewiecki EM, Miyauchi A, Zerbini CAF, Milmont CE, Chen L, Maddox J, Meisner PD, Libanati C, Grauer A. Romosozumab Treatment in Postmenopausal Women with Osteoporosis. New England Journal of Medicine 2016;375(16):1532-1543.

17. Eisman JA, Geusens P, van den Bergh J. Complementarity of Cohort Studies and Randomized Controlled Trials. Journal of bone and mineral research : the official journal of the American Society for Bone and Mineral Research 2019:jbmr.3809.

18. Eisman JA, Geusens P, van den Bergh J. The Emperor's New Clothes: What Randomized Controlled Trials Don't Cover. Journal of bone and mineral research : the official journal of the American Society for Bone and Mineral Research 2018;33(8):1394-1396.

19. Grove EL, Abrahamsen B, Vestergaard P. Heart failure in patients treated with bisphosphonates. Journal of Internal Medicine 2013;274(4):342-350.

20. Abrahamsen B, Eiken P, Brixen K. Atrial fibrillation in fracture patients treated with oral bisphosphonates. Journal of Internal Medicine 2009;265(5):581-592.

21. Rubin KH, Möller S, Choudhury A, Zorina O, Kalsekar S, Eriksen EF, Andersen M, Abrahamsen B. Cardiovascular and skeletal safety of zoledronic acid in osteoporosis observational, matched cohort study using Danish and Swedish health registries. Bone 2020:115296.

22. Sing C-W, Wong AY, Kiel DP, Cheung EY, Lam JK, Cheung TT, Chan EW, Kung AW, Wong IC, Cheung C-L. Association of Alendronate and Risk of Cardiovascular Events in Patients With Hip Fracture. Journal of Bone and Mineral Research 2018;33(8):1422-1434.

23. von Elm E, Egger M, Altman DG, Pocock SJ, Gøtzsche PC, Vandenbroucke JP. Strengthening the reporting of observational studies in epidemiology (STROBE) statement: Guidelines for reporting observational studies. British Medical Journal 2007;335(7624):806-808.

24. Safety and effect of bisphosphonates and other osteoporosis medications in relation to differences in clinical biochemical variables including renal function Odense Bisphosphonate Safety Study. Available at: 
https://open.rsyd.dk/OpenProjects/openProject.jsp?lang=da\&openNo=186. Accessed February 20, 2020.

25. Levey AS, Stevens LA, Schmid CH, Zhang YL, Castro AF, Feldman HI, Kusek JW, Eggers P, van Lente F, Greene T, Coresh J, CKD-EPI (Chronic Kidney Disease Epidemiology Collaboration). A new equation to estimate glomerular filtration rate. Annals of internal medicine 2009;150(9):604-12.

26. Abrahamsen B, Rubin KH, Möller S, Choudhury A, Eriksen EF, Andersen M. Cardiovascular safety of zoledronic acid compared with oral bisphosphonates and untreated population controls: an observational cohort study using Danish and Swedish National Health registries. In: ASBMR 2017. MO0652; 2017:S376.

27. OBSS_Rodriguez_Supplementary_JCEM.docx. Available at: https://figshare.com/articles/OBSS_Rodriguez_Supplementary_JCEM_docx/12435488 . Accessed June 5, 2020.

28. Armitage J, Baigent C, Barnes E, Betteridge DJ, Blackwell L, Blazing M, Bowman L, Braunwald E, Byington R, Cannon C, Clearfield M, Colhoun H, Collins R, Dahlöf B, Davies K, Davis B, de Lemos J, Downs JR, Durrington P, Emberson J, Fellström B, Flather M, Ford I, Franzosi MG, Fulcher J, Fuller J, Furberg C, Gordon D, Goto S, Gotto A, Halls H, Harper C, Hawkins CM, Herrington W, Hitman G, Holdaas H, Holland L, Jardine A, Jukema JW, Kastelein J, Kean S, Keech A, Kirby A, Kjekshus J, Knatterud (deceased) G, Knopp (deceased) R, Koenig W, Koren M, Krane V, Landray MJ, LaRosa J, Lonn E, MacFarlane P, MacMahon S, Maggioni A, Marchioli R, Marschner I, Mihaylova B, Moyé L, Murphy S, Nakamura H, Neil A, Newman C, O'Connell R, Packard C, Parish S, Pedersen T, Peto R, Pfeffer M, Poulter N, Preiss D, Reith C, Ridker P, Robertson M, Sacks F, Sattar N, Schmieder R, Serruys P, Sever P, Shaw J, Shear C, Simes J, Sleight P, Spata E, Tavazzi L, Tobert J, Tognoni G, Tonkin A, Trompet S, Varigos J, Wanner C, Wedel H, White H, Wikstrand J, Wilhelmsen L, Wilson K, Young R, Yusuf S, Zannad F. Efficacy and safety of statin therapy in older people: a meta-analysis of individual participant data from 28 randomised controlled trials. The Lancet 2019;393(10170):407-415.

29. Patntirapong S, Poolgesorn M. Alteration of macrophage viability, differentiation, and function by bisphosphonates. Oral Diseases 2018;24(7):1294-1302.

30. Lucenteforte E, Lombardi N, Vetrano DL, la Carpia D, Mitrova Z, Kirchmayer U, Corrao G, Lapi F, Mugelli A, Vannacci A. Inappropriate pharmacological treatment in older adults affected by cardiovascular disease and other chronic comorbidities: A systematic literature review to identify potentially inappropriate prescription indicators. Clinical Interventions in Aging 2017;12:1761-1778.

31. Black DM, Delmas PD, Eastell R, Reid IR, Boonen S, Cauley JA, Cosman F, Lakatos P, Leung PC, Man Z, Mautalen C, Mesenbrink P, Hu H, Caminis J, Tong K, Rosario-Jansen T, Krasnow J, Hue TF, Sellmeyer D, Eriksen EF, Cummings SR, HORIZON Pivotal Fracture Trial. Once-Yearly Zoledronic Acid for Treatment of Postmenopausal Osteoporosis. New England Journal of Medicine 2007;356(18):1809-1822.

32. Kirchmayer U, Sorge C, Sultana J, Lapi F, Onder G, Agabiti N, Cascini S, Roberto G, Corrao G, Vitale C, Lucenteforte E, Mugelli A, Davoli M, Bartolini C, Bernabei R, Bettiol A, Bonassi S, Caputi AP, Chinellato A, Fini M, Gini R, Giorgianni F, Lombardi N, Rea F, Tari M, Trifirò G, Vannacci A, Vetrano DL. Bisphosphonates and cardiovascular risk in elderly patients with previous cardiovascular disease: a population-based nested case-control study in Italy. Therapeutic Advances in Drug Safety 2019;10:204209861983813. 
33. Boonen S, Orwoll E, Magaziner J, Colón-Emeric CS, Adachi JD, BucciRechtweg C, Haentjens P, Kaufman J-M, Rizzoli R, Vanderschueren D, Claessens F, Sermon A, Witvrouw R, Milisen K, Su G, Lyles KW, HORIZON

Recurrent Fracture Trial. Once-yearly zoledronic acid in older men compared with women with recent hip fracture. Journal of the American Geriatrics Society 2011;59(11):2084-90.

34. Reid IR, Horne AM, Mihov B, Stewart A, Garratt E, Wong S, Wiessing KR, Bolland MJ, Bastin S, Gamble GD. Fracture Prevention with Zoledronate in Older Women with Osteopenia. New England Journal of Medicine 2018;379(25):2407-2416.

35. Cummings SR, McCulloch C. Explanations for the difference in rates of cardiovascular events in a trial of alendronate and romosozumab. Osteoporosis International 2020. doi:10.1007/s00198-020-05379-z.

36. van Geel TACM, Bliuc D, Geusens PPM, Center JR, Dinant GJ, Tran T, van den Bergh JPW, McLellan AR, Eisman JA. Reduced mortality and subsequent fracture risk associated with oral bisphosphonate recommendation in a fracture liaison service setting: A prospective cohort study. PLoS ONE 2018;13(6). doi:10.1371/journal.pone.0198006.

37. Alarkawi D, Ali MS, Bliuc D, Pallares N, Tebe C, Elhussein L, Caskey FJ, Arden NK, Ben-Shlomo Y, Abrahamsen B, Diez-Perez A, Pascual J, Pérez-Sáez MJ, Center JR, Judge A, Cooper C, Javaid MK, Prieto-Alhambra D. Oral Bisphosphonate Use and All-Cause Mortality in Patients With Moderate-Severe (Grade 3B-5D) Chronic Kidney Disease: A Population-Based Cohort Study. Journal of Bone and Mineral Research 2020:jbmr.3961.

38. Choi CK, Kweon S-S, Lee Y-H, Nam H-S, Park K-S, Ryu S-Y, Choi S-W, Kim SA, Shin M-H. Nonlinear association between bone mineral density and all-cause mortality: the Dong-gu study. Osteoporosis international : a journal established as result of cooperation between the European Foundation for Osteoporosis and the National Osteoporosis Foundation of the USA 2018;29(9):2011-2020.

39. Johansson H, Odén A, Kanis J, McCloskey E, Lorentzon M, Ljunggren Ö, Karlsson MK, Orwoll E, Tivesten Å, Ohlsson C, Mellström D. Low bone mineral density is associated with increased mortality in elderly men: MrOS Sweden.

Osteoporosis international : a journal established as result of cooperation between the European Foundation for Osteoporosis and the National Osteoporosis Foundation of the USA 2011;22(5):1411-8.

40. Nguyen ND, Center JR, Eisman JA, Nguyen T v. Bone loss, weight loss, and weight fluctuation predict mortality risk in elderly men and women. Journal of bone and mineral research : the official journal of the American Society for Bone and Mineral Research 2007;22(8):1147-54.

41. Chen W, Simpson JM, March LM, Blyth FM, Bliuc D, Tran T, Nguyen T v, Eisman JA, Center JR. Comorbidities Only Account for a Small Proportion of Excess Mortality After Fracture: A Record Linkage Study of Individual Fracture Types. Journal of Bone and Mineral Research 2018;33(5):795-802. 
Figure 1. Patient flow diagram.

Figure 2. Cumulative hazard plot of oBP users compared to matched non-users 
Table 1. Baseline characteristics

\begin{tabular}{|c|c|c|c|c|c|c|c|}
\hline & & \multicolumn{3}{|c|}{ Total study cohort } & \multicolumn{3}{|c|}{ Propensity score matched sub-cohort } \\
\hline & & oBP users & All non-users & St. Diff. & oBP users & Non-users & St. Diff. \\
\hline & $\mathbf{n}$ & 5,643 & 21,879 & & 2,565 & 4,568 & \\
\hline \multicolumn{2}{|l|}{ Age (years) } & $69[62,77]$ & $51[45,60]$ & 1.612 & $63[57,69]$ & $62[57,69]$ & 0.092 \\
\hline \multicolumn{2}{|l|}{ Women $[\mathrm{n}(\%)]$} & 4564 [80.9] & $16730[76.5]$ & 0.109 & 2118 [82.6] & $3761[82.3]$ & 0.005 \\
\hline \multirow{6}{*}{$\begin{array}{l}\text { BMI }\left[\mathrm{kg} / \mathrm{m}^{2}\right] \& \\
(\mathrm{n}[\%])\end{array}$} & Median [IQR] & $25.9[23.0,29.3]$ & $24.8[22.1,27.6]$ & 0.268 & $24.7[21.9,27.7]$ & $25.1[22.4,28.0]$ & 0.011 \\
\hline & Underweight $<18$ & $263[4.7]$ & $574[2.6]$ & 0.254 & $107[4.2]$ & $223[4.9]$ & 0.014 \\
\hline & Normal 18-25 & $2624[46.5]$ & $8545[39.1]$ & & $1236[48.2]$ & 2015 [44.1] & \\
\hline & Overweight $25-30$ & 1989 [35.2] & 7995 [36.5] & & 858 [33.5] & $1698[37.2]$ & \\
\hline & Obese30-35 & $569[10.2]$ & $3247[14.8]$ & & $252[9.8]$ & $482[10.6]$ & \\
\hline & Very obese $35+$ & $198[3.5]$ & 1518 [6.9] & & $112[4.4]$ & $150[3.3]$ & \\
\hline \multirow{7}{*}{$\begin{array}{l}\text { Charlson index } \\
\text { co-morbidities } \\
(\mathrm{n}[\%])\end{array}$} & 0 & 1656 [29.3] & $16724[76.4]$ & 0.848 & 1144 [44.6] & $2457[53.8]$ & 0.031 \\
\hline & 1 & 1191 [21.1] & $1907[8.7]$ & & $519[20.2]$ & $561[12.3]$ & \\
\hline & 2 & $1003[17.8]$ & $1379[6.3]$ & & 408 [15.9] & $556[12.2]$ & \\
\hline & 3 & 668 [11.8] & $743[3.4]$ & & 204 [8.0] & $382[8.4]$ & \\
\hline & 4 & $435[7.7]$ & $465[2.1]$ & & $123[4.8]$ & 254 [5.6] & \\
\hline & 5 & $230[4.1]$ & 218 [1.0] & & $45[1.8]$ & $124[2.7]$ & \\
\hline & $6+$ & 460 [8.2] & $443[2.0]$ & & $122[4.8]$ & $234[5.1]$ & \\
\hline \multirow{5}{*}{$\begin{array}{l}\text { Fracture history } \\
(\mathrm{n}[\%])\end{array}$} & Any & 2118 [37.5] & $2152[9.8]$ & 0.694 & $754[29.4]$ & $1133[24.8]$ & 0.110 \\
\hline & Hip & 749 [13.3] & $680[3.1]$ & 0.377 & $240[9.4]$ & 373 [8.2] & 0.050 \\
\hline & Spine & 403 [7.1] & $402[1.8]$ & 0.258 & $143[5.6]$ & $222[4.9]$ & 0.029 \\
\hline & Arm & $1145[20.3$ & $1153[5.3]$ & 0.466 & 382 [14.9] & $656[14.4]$ & 0.020 \\
\hline & Humeral & $466[8.3]$ & $497[2.3]$ & 0.274 & $157[6.1]$ & $267[5.8]$ & 0.022 \\
\hline \multirow{4}{*}{$\begin{array}{l}\text { Medication } \\
\text { history (n[\%]) }\end{array}$} & Anti-hypertensive & $2359[41.8]$ & 4719 [21.6] & 0.448 & $733[28.6]$ & 1365 [29.9] & 0.024 \\
\hline & Lipid-lowering & $1355[24.0]$ & $1310[6.0]$ & 0.529 & 315 [12.3] & $582[12.7]$ & 0.010 \\
\hline & Anti-thrombotic & $1530[27.1]$ & $2197[10.0]$ & 0.453 & $391[15.2]$ & $707[15.5]$ & 0.002 \\
\hline & Anti-inflammatory & $2130[37.7]$ & $5719[26.1]$ & 0.251 & 854 [33.3] & $1463[32.0]$ & 0.024 \\
\hline
\end{tabular}




\begin{tabular}{|c|c|c|c|c|c|c|c|}
\hline \multirow{7}{*}{$\begin{array}{l}\text { Renal function } \\
{\left[\mathrm{mL} / \mathrm{min} / 1.73 \mathrm{~m}^{2}\right.} \\
] \text { \& }(\mathrm{n}[\%]])\end{array}$} & Anti-diabetic & $331[5.9]$ & $792[3.6]$ & 0.100 & $101[3.9]$ & $176[3.9]$ & 0.004 \\
\hline & Prednisolone & 1500 [26.6] & $1063[4.9]$ & 0.626 & 365 [14.2] & $609[13.3]$ & 0.026 \\
\hline & Median [IQR] & $83.8[72.3,93.3]$ & $81.4[70.1,90.7]$ & 0.124 & $82.8[70.8,92.3]$ & $80.7[70.5,89.2]$ & 0.062 \\
\hline & Normal $>=90$ & $1516[27.9]$ & $7350[34.7]$ & 0.128 & 797 [32.5] & 1063 [24.1] & 0.087 \\
\hline & Mild 60-89 & $3265[60.2]$ & $11723[55.4]$ & & $1376[56.0]$ & 2911 [65.9] & \\
\hline & Moderate 30-59 & 607 [11.2] & $1880[8.9]$ & & $251[10.2]$ & $417[9.4]$ & \\
\hline & Severe impairment 15-29 & $19[0.4]$ & $103[0.5]$ & & $10[0.4]$ & $12[0.3]$ & \\
\hline \multirow{3}{*}{$\begin{array}{l}\text { CVD history } \\
(\mathrm{n}[\%])\end{array}$} & Kidney failure $<15$ & $21[0.4]$ & $112[0.5]$ & & $21[0.9]$ & $13[0.3]$ & \\
\hline & & $204[3.6]$ & $1869[8.5]$ & 0.207 & $217[4.8]$ & $90[3.5]$ & 0.064 \\
\hline & Total hip BMD & $0.714[0.631,0.803]$ & $0.817[0.721,0.918]$ & 0.723 & $0.734[0.658,0.817]$ & $0.731[0.639,0.829]$ & 0.002 \\
\hline \multirow{8}{*}{ Bone health } & Total hip T-score & $-1.9[-2.5,-1.2]$ & $-1.1[-1.8,-0.3]$ & 0.735 & $-1.7[-2.3,-1.1]$ & $-1.7[-2.5,-1.0]$ & 0.002 \\
\hline & Osteoporosis (total hip) & $1515[26.8]$ & $2345[10.7]$ & 0.465 & $513[20.0]$ & 1146 [25.1] & 0.086 \\
\hline & Femoral neck BMD & $0.598[0.532,0.670]$ & $0.677[0.598,0.766]$ & 0.669 & $0.610[0.549,0.681]$ & $0.612[0.537,0.695]$ & 0.054 \\
\hline & Femoral neck T-score & $-2.2[-2.8,-1.6]$ & $-1.6[-2.2,-0.8]$ & 0.674 & $-2.1[-2.7,-1.5]$ & $-2.1[-2.8,-1.4]$ & 0.053 \\
\hline & $\begin{array}{l}\text { Osteoporosis (femoral } \\
\text { neck) }\end{array}$ & $2275[40.3]$ & $4109[18.8]$ & 0.492 & $886[34.5]$ & $1650[36.1]$ & 0.024 \\
\hline & Lumbar spine BMD & $0.802[0.727,0.896]$ & $0.911[0.815,1.023]$ & 0.705 & $0.796[0.732,0.881]$ & $0.852[0.752,0.964]$ & 0.355 \\
\hline & Lumbar spine T-score & $-2.3[-2.9,-1.5]$ & $-1.3[-2.1,-0.3]$ & 0.720 & $-2.4[-2.9,-1.7]$ & $-1.7[-2.7,-0.7]$ & 0.437 \\
\hline & $\begin{array}{l}\text { Osteoporosis (lumbar } \\
\text { spine) }\end{array}$ & 2422 [42.9] & 3715 [17.0] & 0.559 & $1171[45.7]$ & 1318 [28.9] & 0.406 \\
\hline
\end{tabular}


Table 2. Incidence rates for the primary study outcome [hospitalization for any CV event] in propensity score-matched sub-cohorts analysed on intention-to-treat and per-protocol bases

\begin{tabular}{|c|c|c|c|c|c|c|}
\hline Analysis & & Events (\%) & Follow-up years [IQR] & Person-years at risk & $\begin{array}{c}\text { Incidence rate }[95 \% \mathrm{CI}] \text { per } 1000 \\
\text { person-years at risk }\end{array}$ & Incidence rate ratio $[95 \% \mathrm{CI}]$ \\
\hline \multirow{2}{*}{ Intention-to-treat } & oBP User & 406 & $1.55[0.71,3.04]$ & 5525 & $73.48[66.67,80.98]$ & \multirow{2}{*}{$0.70[0.62,0.79]$} \\
\hline & Control & 837 & $1.87[0.36,4.17]$ & 7991 & $104.73[97.87,112.07]$ & \\
\hline \multirow{2}{*}{ Per-protocol } & oBP User & 314 & $1.17[0.43,2.50]$ & 3655 & $85.90[76.90,95.95]$ & \multirow{2}{*}{$0.82[0.71,0.93]$} \\
\hline & Control & 837 & $1.87[0.36,4.17]$ & 7991 & $104.73[97.87,112.07]$ & \\
\hline
\end{tabular}


Table 3. Effects estimates [Hazard ratios and $95 \%$ confidence intervals] in the propensity-score matched sub-cohorts analysed on an intention-to-treat and per-protocol basis

\begin{tabular}{|c|c|c|c|c|c|c|}
\hline \multirow{2}{*}{$\begin{array}{c}\text { Analysis } \\
\text { Adjustments }\end{array}$} & \multicolumn{3}{|c|}{ Intention-to-treat } & \multicolumn{3}{|c|}{$\underline{\text { Per-protocol }}$} \\
\hline & oBP & oBP \& Pscore & $\begin{array}{c}\text { oBP \& Pscore } \& \\
\text { Unbalanced covariates }\end{array}$ & oBP & oBP \& Pscore & $\begin{array}{c}\text { oBP \& Pscore } \& \\
\text { Unbalanced covariates }\end{array}$ \\
\hline \multicolumn{7}{|l|}{ Hospitalisation outcomes } \\
\hline Any CVD event & $0.74[0.65,0.83]$ & $0.68[0.60,0.77]$ & $0.67[0.58,0.78]$ & $0.90[0.79,1.03]$ & $0.84[0.73,0.96]$ & $0.82[0.70,0.97]$ \\
\hline Atrial fibrillation & $0.67[0.55,0.81]$ & $0.60[0.49,0.74]$ & $0.63[0.49,0.80]$ & $0.79[0.63,0.99]$ & $0.71[0.56,0.89]$ & $0.74[0.56,0.97]$ \\
\hline Cardiac arrhythmia & $0.61[0.41,0.89]$ & $0.61[0.41,0.90]$ & $0.66[0.41,1.05]$ & $0.80[0.52,1.23]$ & $0.80[0.52,1.24]$ & $0.89[0.53,1.50]$ \\
\hline Myocardial infarction & $0.92[0.65,1.30]$ & $0.86[0.61,1.23]$ & $0.90[0.59,1.38]$ & $1.04[0.70,1.55]$ & $0.98[0.65,1.48]$ & $1.03[0.63,1.68]$ \\
\hline Heart failure & $0.76[0.59,0.98]$ & $0.68[0.52,0.89]$ & $0.65[0.47,0.90]$ & $0.97[0.74,1.29]$ & $0.90[0.67,1.20]$ & $0.84[0.59,1.20]$ \\
\hline Stroke & $0.60[0.47,0.77]$ & $0.56[0.44,0.72]$ & $0.46[0.34,0.63]$ & $0.82[0.62,1.07]$ & $0.76[0.58,1.00]$ & $0.61[0.44,0.86]$ \\
\hline Ischaemic stroke & $0.64[0.50,0.82]$ & $0.60[0.47,0.78]$ & $0.50[0.37,0.69]$ & $0.86[0.66,1.14]$ & $0.81[0.61,1.07]$ & $0.66[0.47,0.93]$ \\
\hline Atherosclerotic & $0.45[0.32,0.63]$ & $0.42[0.29,0.60]$ & $0.35[0.23,0.55]$ & $0.63[0.43,0.93]$ & $0.59[0.40,0.88]$ & $0.51[0.31,0.82]$ \\
\hline Aneurysm & $0.55[0.45,0.69]$ & $0.53[0.42,0.66]$ & $0.57[0.44,0.74]$ & $0.72[0.57,0.92]$ & $0.69[0.54,0.88]$ & $0.75[0.56,1.00]$ \\
\hline Peripheral artery disease & $0.73[0.49,1.10]$ & $0.71[0.47,1.08]$ & $0.65[0.39,1.09]$ & $0.95[0.60,1.51]$ & $0.92[0.57,1.47]$ & $0.88[0.49,1.55]$ \\
\hline \multicolumn{7}{|l|}{ Mortality outcomes } \\
\hline Any CVD death & $0.55[0.36,0.86]$ & $0.48[0.31,0.75]$ & $0.50[0.29,0.87]$ & $0.44[0.25,0.77]$ & $0.36[0.20,0.65]$ & $0.37[0.19,0.74]$ \\
\hline Stroke death & $0.45[0.19,1.07]$ & $0.37[0.15,0.92]$ & $0.37[0.12,1.09]$ & $0.39[0.13,1.16]$ & $0.33[0.11,1.01]$ & $0.45[0.13,1.53]$ \\
\hline Myocardial infarction & $0.64[0.27,1.52]$ & $0.56[0.23,1.35]$ & $0.53[0.19,1.48]$ & $0.67[0.24,1.87]$ & $0.52[0.18,1.50]$ & $0.47[0.13,1.63]$ \\
\hline All-cause & $0.59[0.49,0.70]$ & $0.45[0.37,0.54]$ & $0.35[0.28,0.44]$ & $0.38[0.30,0.49]$ & $0.28[0.22,0.37]$ & $0.23[0.17,0.31]$ \\
\hline
\end{tabular}

Unbalanced covariates: lumbar spine BMD, osteoporosis in the spine, prior fracture at any site. Pscore = propensity score 
Table 4. Effects estimates [Hazard ratios and $95 \%$ confidence intervals] for control outcomes in propensity-score matched sub-cohorts analysed on an intention-to-treat and perprotocol basis

\begin{tabular}{|c|c|c|c|c|}
\hline \multirow[b]{2}{*}{ Adjustments } & \multicolumn{4}{|c|}{ Propensity score matched sub-cohort } \\
\hline & Events (user/control) & oBP & oBP \& Pscore & oBP \& Pscore \& Unbalanced covariates \\
\hline \multicolumn{5}{|c|}{ Intention-to-treat analysis } \\
\hline Inguinal hernia surgery & $75 / 164$ & $0.90[0.69,1.19]$ & $0.91[0.69,1.21]$ & $0.94[0.67,1.32]$ \\
\hline Ingrown toenail & $4 / 1$ & $8.32[0.92,74.7]$ & $9.71[1.05,89.25]$ & $8,73[0.77,98.11]$ \\
\hline Osteoporotic fracture & $247 / 630$ & $0.81[0.70,0.94]$ & $0.76[0.65,0.88]$ & $0.26[0.22,0.33]$ \\
\hline \multicolumn{5}{|l|}{ Per-protocol analysis } \\
\hline Inguinal hernia surgery & $75 / 164$ & $1.15[0.87,1.51]$ & $1.16[0.87,1.55]$ & $1.19[0.85,1.67]$ \\
\hline Ingrown toenail & $4 / 1$ & $11.12[1.22,100.77]$ & $12.98[1.40,120.64]$ & $12.38[1.08,141.43]$ \\
\hline Osteoporotic fracture & $219 / 630$ & $0.92[0.78,1.07]$ & $0.85[0.72,1.00]$ & $0.31[0.25,0.38]$ \\
\hline
\end{tabular}

Unbalanced covariates: lumbar spine BMD, osteoporosis in the spine, prior fracture at any site. Pscore = propensity score 